\title{
Exposição ao benzeno em postos de revenda de combustíveis no Brasil: Rede de Vigilância em Saúde do Trabalhador (VISAT)
}

\author{
Exposure to benzene in gas stations in Brazil: occupational health \\ surveillance (VISAT) network
}

\author{
Maria Juliana Moura-Correa ${ }^{1}$ \\ Alexandre José Ribeiro Jacobina ${ }^{2}$ \\ Simone Alves dos Santos ${ }^{3}$ \\ Regina Dal Castel Pinheiro ${ }^{4}$ \\ Marco Antônio Carneiro Menezes ${ }^{5}$ \\ Andrea Marques Tavares ${ }^{6}$ \\ Nanci Ferreira Pinto ${ }^{7}$
}

Abstract The scope of this article is to present the surveillance network's experience of exposure to benzene in gas stations, describing its components developed in the Unified Health System. It is a report and analysis of the experience of monitoring and its impact on the health of gas station workers in six Brazilian states. It presents the prospect of action, operated by the circulation of information, national meetings, discussions of specific strategies and shared experiences, methodologies and common tools. Inspection procedures, individual assessments and training are described. Risk situations and occupational and environmental exposure were identified in 1,311 gas stations and 564 workers were assessed. Forecourt attendants, superintendents and measuring, offloading and testing employees were all exposed. The integrated and complementary features of this surveillance, implemented in the occupational and environmental areas, suggest intervention in a broader and network-linked territory. The intervention would transcend fragmentation between individual and collective practices, services and education, becoming an example of action for assessment and mitigation of the impact on health workers, the articulation of action in health, the environment and intersectorial connection.

Key words Occupational health, Benzene, Surveillance, Gas stations
Resumo $O$ objetivo deste artigo é apresentar a experiência da rede de vigilância da exposição ao benzeno em Postos de Revenda de Combustíveis, descrevendo seus componentes, desenvolvida no Sistema Único de Saúde. Relato e análise de experiência de vigilância e seus impactos à saúde dos trabalhadores de postos de combustiveis em seis estados do Brasil. Apresenta a perspectiva de ação operada pela circulação de informações, encontros nacionais, discussões de estratégias particulares e compartilhadas das experiências, metodologias e instrumentos comuns. São descritos procedimentos de inspeção, avaliações individuais e formação. Foram identificadas situações de risco e exposições ocupacionais e ambientais em 1.312 postos e 564 trabalhadores foram avaliados. Estavam expostos frentistas, chefes de pista e encarregados de medição, descarga e testes. As características integradas e complementares desta vigilância, articuladas às áreas ocupacional e ambiental, contemplam a intervenção a territórios ampliados e conectados em rede. A intervenção supera as fragmentações entre práticas individuais e coletivas e entre serviços e ensino, tornando-se um exemplo de ação para avaliação e redução do impacto à saúde em trabalhadores, pela articulação da ação sanitária, ambiental e de conexão intersetorial.

Palavras-chave Saúde do Trabalhador, Benzeno, Vigilância, Postos de Revenda de Combustiveis

\footnotetext{
Fundação Oswaldo Cruz.
${ }^{6}$ Centro de Referência

Regional em Saúde do

Trabalhador, Secretaria

Campinas.

${ }^{7}$ Centro Estadual de Saúde do Trabalhador, Secretaria de Estado da Saúde do Paraná.
} 


\section{Introdução}

O benzeno é um hidrocarboneto aromático, de exposição ubíqua/contaminante universal, no meio ambiente e nos processos de trabalho. Esta substância está presente na composição da gasolina e em outros compostos orgânicos produzidos pela indústria química e petroquímica ${ }^{1}$. Por tratar-se de uma substância carcinogênica e hematotóxica, é responsável pela ocorrência de doenças do sistema hematopoiético, entre elas, a leucemia ${ }^{2}$. Devido aos efeitos à saúde, esta exposição é alvo de Vigilância em Saúde (VS), em todo o mundo.

No Brasil, a VISAT (VISAT), componente da VS do Sistema Único de Saúde (SUS), ocupa papel central na intervenção sobre os fatores de riscos e os determinantes dos agravos à saúde dos trabalhadores ${ }^{3}$.

As exposições químicas, entre elas o benzeno, são prioridades para a VISAT, por parte da Rede Nacional de Atenção Integral à Saúde do Trabalhador (Renast), e instituídas por normas ${ }^{4}$. Em alguns estados do país, as coordenações de Saúde do Trabalhador, os centros de Referência em Saúde do Trabalhador estaduais e regionais e as VS das secretarias estaduais ou municipais de Saúde do SUS incorporam essa vigilância em Postos de Revenda de Combustíveis no planejamento de ações de VISAT ${ }^{5}$. Destaca-se os trabalhos realizados no Centro de Referência Regional em Saúde do Trabalhador de Campinas - CEREST/SP e nas secretarias de Saúde dos estados da BA, PR, RS, SC e SP. A exposição neste ambiente de trabalho também foi incorporada na agenda da Comissão Nacional Permanente do Benzeno (CNPBz), coordenada pelo Ministério do Trabalho e Emprego (MTE) ${ }^{6}$ e das comissões Regionais do Benzeno, em especial da Comissão Regional do Paraná.

Entretanto, ainda persistem lacunas sobre o modelo de intervenção, as condições de saúde e trabalho e a gravidade desta exposição ocupacional no Brasil. Exemplo disso são os métodos para VISAT, que são múltiplos e dependem do domínio de determinada disciplina sobre o objeto de investigação. No âmbito dessa complexa exposição ocupacional, diversas disciplinas têm contribuído para análise e investigação da exposição. Porém, a integralidade da intervenção em VISAT - definição normativa estratégica - ainda não está plenamente instituída no SUS. Nesse sentido, o Sistema Nacional de Vigilância em Saúde (SNVS) tem apresentado os marcos teóricos e conceituais dos componentes da VS em seus aspectos sanitário, epidemiológico, da promoção da saúde, prevenção e controle de doenças e de agravos à saúde ${ }^{7}$. Paim ${ }^{8}$ evidencia que esses componentes se estruturam em três níveis de ação de vigilância: controle de causa, dos riscos e dos danos. Ademais, Machado ${ }^{9}$, ao caracterizar a VISAT como uma área de promoção da saúde, destaca que é uma prática que requer ação transdisciplinar e atuação inter e intrassetorial. Portanto, para fins da VISAT é primordial que estes componentes se articulem para uma intervenção de impacto. No entanto, a integralidade desses componentes é, ainda, um desafio à organização da vigilância no SUS.

No campo das exposições ao benzeno, por exemplo, diversos ambientes de trabalho e grupos ocupacionais estão submetidos a essa substância. Entre eles, os trabalhadores de postos de combustíveis. Estudos ${ }^{10,11}$ têm alertado para a importância da vigilância deste grupo ocupacional em virtude das concentrações de benzeno em 1 ppm no ar e da ausência de normatização, fiscalização e monitoramento. O número de Trabalhadores em PRC no Brasil é expressivo. O censo de 2010 registrou 184.733 frentistas ${ }^{12}$, distribuídos em 39.450 PRC, em todo o país ${ }^{13}$.

Este artigo tem como objetivo apresentar a experiência de VISAT em PRC, descrevendo os componentes da intervenção, desenvolvidos em rede pelos CEREST do SUS, no Brasil.

\section{Materiais e métodos}

Este estudo descritivo relata a experiência da rede de VISAT em PRC em seis estados do Brasil, entre 2004 e 2014: 1. Bahia; 2. Paraná; 3. Rio Grande do Sul; 4. Rio de Janeiro; 5. Santa Catarina; e 6. São Paulo.

A população é composta por trabalhadores que realizam atividades em PRC. Na maioria dos estados, a seleção dos PRCs foi feita por amostra de conveniência, à exceção de São Paulo, cujo critério foi o de áreas contaminadas.

Para a avaliação dos ambientes de trabalho foi utilizado o instrumento denominado Roteiro de Inspeção Sanitária de Ambientes e Processos de Trabalho, produzido pelo Centro Estadual de Referência em Saúde do Trabalhador da Bahia (Cesat/BA). Para as demais ações, cada serviço utilizou instrumentos próprios.

Os dados de avaliação dos trabalhadores são oriundos da assistência e estudos, que foram integrados a essa ação em rede. No momento, estes dados não serão detalhados, mas compõem o total de trabalhadores assistidos pela ação VISAT em PRC. 


\section{Descrição do percurso metodológico}

\section{O contexto da experiência de VISAT}

A proposição da estratégia da VISAT em PRC teve início em 2005, como resultado da experiência e conhecimento científico dos técnicos do governo pertencentes ao grupo tripartite do Acordo Nacional do Benzeno (ANB). A proposta também teve como base experiências dos serviços de Saúde do Trabalhador, que já promoviam ações em vigilância nos PRCs, a exemplo dos estados de São Paulo (2002) $)^{14}$ e Bahia $(2004)^{15}$.

Em 2009, é constituída a rede de VISAT em PRC, a partir da articulação nacional viabilizada pelo projeto "Vigilância da Exposição a Solventes nos Postos de Combustíveis do Brasil", Fiocruz -DSAST-SVS-MS, proposto em 2008 e executado em 2009-2010 unificada pela priorização da inspeção nos ambientes de trabalho em seis estados. Sua operacionalização se efetiva na pactuação entre os serviços, unificação do instrumento de inspeção e formação fundamentada, no caso da exposição ocupacional ao benzeno. Em uma ação nacional, a perspectiva interdisciplinar $\mathrm{e}$ multiprofissional de integração em rede se viabiliza pela autonomia de organização local, na medida em que esta permite o desenvolvimento de ações diferenciadas com base nos componentes de VS, considerando a capacidade instalada e o perfil dos serviços, articulados à produção do conhecimento por meio da Fiocruz. A dinâmica processual dessa metodologia exigiu definições e momentos de sínteses, pactuações e avaliações com os diferentes atores que compõem a rede, $\mathrm{o}$ que levou à constituição do Centro de Estudos da Saúde do Trabalhador e Ecologia Humana da Es-

cola Nacional de Saúde Pública Sergio Arouca, da Fundação Oswaldo Cruz (Cesteh/Ensp/Fiocruz), enquanto ponto central da rede. Essa definição estrutural e de articulação entre os pontos da rede tornou-se fundamental para a continuidade da VISAT e da funcionalidade do processo e do método de trabalho que integram ensino, pesquisa, serviço e trabalhadores. O conjunto dessas experiências retroalimenta todos os serviços e equipes participantes desta rede de VISAT, instituições de ensino e representação da sociedade civil sem, no entanto, prescindir de sua idêntica reprodução em todos os serviços e/ou estados. Esse conjunto se completa pela apropriação dos resultados das ações multiprofissional e interinstitucional sobre o objeto de intervenção, transformando-se em parte da totalidade que integra, para a compreensão dessa complexa exposição e seus efeitos à saúde dos trabalhadores.

As especificidades das experiências nos serviços de Saúde do Trabalhador levam em consideração o acúmulo histórico da prática e a capacidade instalada. Nos estados de $\mathrm{SP}^{14}, \mathrm{BA}^{15}$, $\mathrm{PR}^{16} \mathrm{e} \mathrm{SC}^{17}$, as ações em rede de VISAT em PRC iniciaram com o processo de formação em vigilância da exposição ao benzeno e do projeto de intervenção, entre 2005 a 2010. Em São Paulo, na região de abrangência do CEREST/Campinas ${ }^{18}$, as ações foram desencadeadas a partir do evento sentinela - diagnóstico de leucopenia de um frentista, para o qual foi integrado um conjunto de estratégias interinstitucionais entre os órgãos da saúde e do trabalho, envolvendo as áreas de vigilância de Saúde do Trabalhador, Sanitária e Ambiental. No Rio Grande do Sul ${ }^{19}$ e Rio de Janeiro $^{20}$, as ações se estruturaram pelo eixo da formação, alicerçada em estudos e pesquisas em parcerias intrasetoriais e interinstitucionais.

\section{A estratégia de ação de rede de VISAT e seus componentes}

A vigilância, orientada pela formação de redes intrasetoriais e interinstitucionais em torno de um projeto de intervenção, tem sido a proposta de ação transversal, agregadora de expertises individuais e institucionais em torno de um problema complexo que requer a união de especialidades, para obtenção de um maior impacto na intervenção e proteção à saúde ${ }^{21}$.

Nesse aspecto, com vistas a potencializar a integração das ações em rede, embasados nos marcos teóricos que estruturam a VISAT, foram organizados os seguintes componentes estruturantes da vigilância: 


\section{Ensino em serviço para vigilância da exposição ao benzeno}

Com base nas premissas de formação para VISAT destacado por Vasconcelos et al..$^{22}$, o programa de capacitação para VISAT em PRC foi concebido considerando os princípios do SUS e o formato participativo, onde técnicos e trabalhadores do ramo produtivo foram integrados às etapas de capacitação e inspeção dos locais de trabalho.

Os cursos, estruturados pelo eixo central da formação-ação, expandiram seus limites geográficos pela incorporação dos seis estados, ampliando seu alcance territorial pela dimensão da atuação dos serviços em níveis locais, regionais, estaduais e, também, o espaço nas universidades compondo, desta forma, o cenário de exposição e intervenção em nível nacional. Os cursos tiveram o objetivo de aprimorar habilidades e competências técnicas para investigação e intervenção com foco na exposição ao benzeno. Para isso, em todos os estados da rede de VISAT em PRC ${ }^{14-20}$, foram realizados cursos, oficinas e seminários envolvendo aspectos teóricos sobre a exposição para fins de vigilância por meio de práticas orientadas para a observação de riscos nos ambientes e preenchimento da Ficha de Inspeção em PRC.

\section{Pesquisa sobre a exposição ocupacional ao benzeno}

As pesquisas desenvolvidas em parceria interinstitucional com os serviços do SUS e instituições de ensino e pesquisa ocorrem especificamente nos estados do Rio Grande do Sul ${ }^{19}$ e Rio de Janeiro ${ }^{20}$. Essas envolveram estudos sobre indicadores biológicos de genotoxicidade, susceptibilidade, ototoxicidade e exposição ambiental ${ }^{23,24}$.

Também contribuíram para essa linha de investigação os estudos desenvolvidos junto à pós-graduação do departamento de Farmácia da Universidade Federal de Minas Gerais (UFMG) ${ }^{25}$.

Vigilância em rede da exposição nos processo e ambiente de trabalho

A vigilância dos ambientes de trabalho foi planejada nos seis estados, seguindo o roteiro de inspeção aprimorado pelos demais órgãos participantes do projeto. No roteiro, constam 14 blocos de questões, num total de 156 itens, sobre fatores de riscos ocupacionais, perfil sóciodemográfico e medidas de prevenção e controle coletivo e individual para avaliação qualitativa e quantitativa. Além desses, outros dados podem ser inseridos mediante observações dos técnicos e percepção dos trabalhadores.
Em relação às modalidades e instrumentos administrativos utilizados nas vigilâncias em cada estado, estas compõem distintas experiências de apreensão da realidade e intervenção.

No Rio Grande do Sul ${ }^{19}$, essa experiência de vigilância desencadeou a adoção do instrumento de notificação recomendatória dos técnicos dos CEREST, em parceria com o Ministério Público do Trabalho (MPT), transformando-se em ação coletiva para todos os demais PRC da região, tomando como exemplos os achados das avaliações realizadas nos ambientes e vistorias com os trabalhadores. Esta modalidade de intervenção permitiu ampliar as recomendações aos demais PRCs neste estado, uma vez que o ambiente e processo de trabalho são semelhantes na maioria dos PRCs.

$\mathrm{Na} \mathrm{Bahia}^{15}$, o procedimento de inspeção compreendeu as etapas de mapeamento dos PRCs e o preenchimento do roteiro que subsidiam a emissão das notificações por parte dos técnicos do CESAT que, em geral, abrangem em torno de 40 recomendações. Os relatórios foram individualizados para cada PRC inspecionado e, quando não cumpridas essas recomendações, os mesmos são encaminhados ao MPT, procedimento que fundamenta a abertura do instrumento de Termo de Ajustamento de Conduta (TAC). O Programa de VISAT em PRC, coordenado pelo Centro de Vigilância Sanitária da Secretaria de Saúde do Estado de São Paulo (SES/SP) ${ }^{14}$, compreendeu ações de controle de risco pela intervenção sanitária, visando à proteção da saúde dos trabalhadores e da população do entorno. Neste estado, a estratégia adotada foi a articulação intrasetorial entre a Vigilância Sanitária (VISA), Vigilância Ambiental (VISAM) e órgão do Meio Ambiente. Em Santa Catarina ${ }^{17}$, orientados pela ação sanitária, foi realizado um mapeamento dos PRCs pelos CEREST (Lages, Criciúma, Chapecó, Joinville, Florianópolis) e VISA (regionais e municipais de SC) e emitidos autos de intimação para o cumprimento de recomendações à saúde e segurança. Além desses procedimentos administrativos e suas etapas normativas, foi associada uma ação coletiva por meio de uma reunião pública com os proprietários de PRC e sindicatos de trabalhadores para apresentação dos resultados e pactuação das medidas de melhorias nas condições de trabalho.

Esses instrumentos administrativos, com intervenções e atuações distintas, unificam-se pela utilização do mesmo roteiro de inspeção para avaliação dos ambientes e da saúde dos trabalhadores que subsidiam a notificação. Em todos os estados, a ação foi integrada com os CEREST re- 


\section{Prevenção da exposição ocupacional ao benzeno}

Em algumas regiões foram realizadas iniciativas de campanhas prevencionistas ${ }^{14,17,18}$ por meio de instrumentos de educação e divulgação dos riscos: folders, cartilhas, produção de vídeo e campanhas educativas para abastecimento automático, com a participação dos proprietários de PRC, trabalhadores e população.

\section{A participação dos trabalhadores}

Essa perspectiva de atuação segue a concepção definida por Santos ${ }^{26}$ de que determinada distribuição de pessoas ocorre pela produção sobre o território. A garantia dessa reprodução conceitual para a prática requer a construção de um processo pedagógico de produção do conhecimento validado entre trabalhadores e técnicos, conforme preconizado por Laurell e Noriega ${ }^{27}$.

Para isso, de forma distinta e criativa, as experiências nos estados apostam na inserção dos trabalhadores e sindicatos na participaram das ações, desde o processo de formação dos técnicos nas inspeções dos ambientes de trabalho e de reunião com os trabalhadores e empresários, até a elaboração de materiais educativos. É como na experiência do Rio de Janeiro, que apresenta sindicatos e trabalhadores integrados no processo de pesquisa, divulgação dos resultados, debates e avaliações por meio de seminários com todos os participantes da rede.

\section{As bases epidemiológicas da intervenção}

Nesta ação em rede, as bases epidemiológicas ainda estão em processo de construção. A concepção em desenvolvimento fundamenta-se pela constituição da vigilância epidemiológica definida por Santana e Ferrite ${ }^{28}$ enquanto etapas que envolvem, além da tradicional coleta de dados, processamento e análise, as recomendações de medidas e execução de ações de controle e, também, a avaliação e efetividade da execução, que requerem a ampla divulgação para os trabalhadores interessados na ação. Portanto, da produção de informação para a ação. 


\section{Resultados}

\section{A dimensão do problema e a ação de VISAT}

Em 2012, no Brasil ${ }^{13}$, os trabalhadores expostos estavam distribuídos em um contingente de 39.450 PRCs operando no país. A concentração é maior na região Sudeste $\left(\mathrm{n}_{\mathrm{PRC}}=16.182 ; 41 \%\right)$, seguida pelas regiões Nordeste $\left(\mathrm{n}_{\text {PRC }}=8.952\right.$; $22,7 \%)$, Sul ( $\left.\mathrm{n}_{\mathrm{PRC}}=8.105 ; 20,5 \%\right)$, Centro-Oeste $\left(\mathrm{n}_{\mathrm{PRC}}=3.381 ; 8,5 \%\right)$ e Norte $\left(7,2 \% ; \mathrm{n}_{\mathrm{PRC}}=2.830\right)$. O consumo na região Sudeste é superior a quase duas vezes ao das regiões Nordeste e Sul.

As ações em rede de vigilância em PRC desenvolvidas representam $55 \%\left(\mathrm{n}_{\mathrm{PRC}}=21.715\right)$ do processo produtivo no Brasil, em 2012. Os PRC ${ }^{13}$ estão distribuídos assimetricamente entre os estados: Bahia $\left(\mathrm{n}_{\mathrm{PRC}}=2.323 ; 5,9 \%\right)$; Paraná $\left(\mathrm{n}_{\mathrm{PRC}}\right.$ $=2.823 ; 7,2 \%)$; Rio Grande do Sul $\left(\mathrm{n}_{\mathrm{PRC}}=3.125\right.$; 7,9\%); Rio de Janeiro ( $\left.\mathrm{n}_{\mathrm{PRC}}=2.230 ; 5,7\right)$; Santa Catarina $\left(\mathrm{n}_{\mathrm{PRC}}=2.157 ; 5,5 \%\right)$; e São Paulo $\left(\mathrm{n}_{\mathrm{PRC}}\right.$ $=9.057 ; 23,0 \%)$.

\section{A população de expostos ao benzeno em PRC}

Além da população trabalhadora diretamente exposta pelo processo de trabalho nas atividades em PRC - nas quais os dados do censo 2010 apontam para 184.733 frentistas $^{12}$-, também estão submetidos a essa exposição ocupacional e ambiental outros tipos de ocupações da área de trabalho e os residentes do entorno. Essa população de expostos é ainda maior, se for considerada a circulação diária de pessoas, a localização dos estabelecimentos em áreas densamente povoadas e os serviços oferecidos que aumentam a população potencialmente exposta ao benzeno em PRC.

\section{A qualificação dos técnicos em VISAT em PRC}

A formação para VISAT em PRC, segundo registros em relatórios ${ }^{14-20}$ apontam para formação de aproximadamente 1.870 trabalhadores em cursos, seminários e oficinas, no período. Especificamente, para o desenvolvimento desta VISAT em PRC, foram capacitados um total de 1.106 técnicos de CEREST, Unidades Básicas de Saúde e fiscais das vigilâncias sanitárias municipais e regionais. Desses, 524 pertenciam ao estado da Bahia, 473 do Rio Grande do Sul, 257 de Santa Catarina, dentre outros. A ação em rede e a formação descentralizada levaram os técnicos de vigilância do CESAT/BA e do CEREST/Campinas a assumirem o papel de ponto focal para qualificação da intervenção e uso do roteiro de inspeção em nove estados do país (SP, RS, RN, CE, AM, AC, SE, PR, GO) ${ }^{15,18}$.

\section{A ação de VISAT em PRC no Brasil}

A VISAT em PRC abrangeu um total de 16.440 trabalhadores (ações pontuais e contínuas) e 1.312 locais de trabalho em PRC em 193 municípios de seis unidades federadas do Brasil, entre 2004 a 2014. Dessas inspeções, foram emitidas 431 notificações. Avaliações clínicas, toxicológicas e audiológicas foram realizadas em 564 trabalhadores de PRC de cinco municípios (Campinas, Ijuí, Santa Cruz do Sul, Santa Maria e Rio de Janeiro), entre 2010 e 2013 (Tabela 1). Das 187 VISAT em PRC realizadas na Bahia, $54 \%\left(n_{\mathrm{PRC}}=100\right)$ eram da capital do Estado (Figura 1). Em Santa Catarina, 621 VISAT em PRC foram distribuídas em 31\% $(\mathrm{n}=91)$ dos municípios do estado $(\mathrm{n}=295)$. Os municípios com maior número de postos foram a capital catarinense, com $12 \%$ dos PRCs vistoriados $\left(\mathrm{n}_{\mathrm{PRC}}=75\right)$, seguido do município de Joinvile $\left(10 \% ; \mathrm{n}_{\mathrm{PRC}}=60\right)$. Nos demais 489 municípios do estado, as vistorias variaram de 1 a 10 PRCs (278) e 11 a 44 PRC (211) (Figura 2).

No âmbito da ação sanitária, a VISAT produziu um conjunto de dados que ainda não foram analisados em sua totalidade. Entretanto, as análises parciais apontam para uma realidade de exposição contínua, por conta da ausência de controle das fontes de escape de vapores de combustíveis no ar durante o processo de abastecimento de veículos, na descarga de caminhões tanques e por vazamentos nas tubulações, solo e água. Além disso, estão presentes neste processo de trabalho outros riscos associados a substâncias químicas, além dos compostos da gasolina como, por exemplo, álcool, diesel e gás. Agrava essa situação a ausência de treinamento dos trabalhadores e de informações sobre o risco, acompanhada por inexpressivo fornecimento de equipamento de proteção individual e baixa realização de exames periódicos específicos para exposição ao benzeno. Além dos resultados obtidos pela aplicação dos roteiros de inspeção, os grupos com trabalhadores apontam questões relativas à violência, assaltos, atropelamento e agressões de clientes, principalmente pelas características do local de trabalho, com grande circulação de pessoas. 
Tabela 1. Distribuição do número de vigilâncias e assistência em trabalhadores de Postos de Revenda de Combustíveis por seis Unidades Federadas. Brasil, 2004 a 2014.

\begin{tabular}{|c|c|c|c|c|c|c|c|c|}
\hline \multirow{4}{*}{$\begin{array}{l}\text { Unidades } \\
\text { Federadas }\end{array}$} & \multirow{4}{*}{ CEREST } & \multirow{4}{*}{$\begin{array}{c}\text { Município } \\
\mathrm{N}^{\mathrm{o}}\end{array}$} & \multicolumn{5}{|c|}{ Ação de VISAT } & \multirow{4}{*}{$\begin{array}{c}\frac{\text { Assistência }}{\text { Avaliações }} \\
\text { individuais } \\
\begin{array}{c}\text { Trab } \\
\mathrm{N}^{\circ}\end{array}\end{array}$} \\
\hline & & & \multicolumn{2}{|c|}{$\begin{array}{l}\text { Fase Piloto } \\
\text { ou Pontual }\end{array}$} & \multirow{3}{*}{$\begin{array}{c}\begin{array}{c}\text { Continua/ } \\
\text { integral }\end{array} \\
\text { PRC } \\
\mathrm{N}^{\mathbf{0}}\end{array}$} & \multirow{3}{*}{$\begin{array}{c}\text { Trab } \\
\text { abrangidos } \\
\text { VISAT } \\
\mathrm{N}^{\circ}\end{array}$} & \multirow{3}{*}{$\begin{array}{c}\begin{array}{c}\text { Emissão de } \\
\text { notificação }\end{array} \\
\text { PRC } \\
\mathrm{N}^{\circ}\end{array}$} & \\
\hline & & & PRC & Trab & & & & \\
\hline & & & $\mathbf{N}^{\circ}$ & $\mathrm{N}^{\mathrm{o}}$ & & & & \\
\hline \multirow{10}{*}{$\mathrm{BA}^{\mathrm{a}}$} & Estadual-CESAT & 3 & 4 & - & 126 & 1.890 & 126 & - \\
\hline & C. do Coité & 2 & 7 & 105 & - & - & 7 & - \\
\hline & Camaçari & 1 & 4 & 60 & - & - & 4 & - \\
\hline & Feira de Santana & 1 & - & - & 19 & 285 & 19 & - \\
\hline & Itaberaba & 1 & - & - & 10 & 150 & 10 & - \\
\hline & Itabuna & 1 & 4 & 60 & - & - & 4 & - \\
\hline & Juazeiro & 1 & 1 & 15 & - & - & 1 & - \\
\hline & Sto Ant. Jesus & 1 & 2 & 30 & - & - & 1 & - \\
\hline & Teixeira Freitas & 1 & 1 & 15 & - & - & 1 & - \\
\hline & Vit Conquista & 1 & - & - & 9 & 135 & 9 & - \\
\hline Subtotal & & 13 & 23 & 285 & 164 & 2.460 & 182 & - \\
\hline $\mathbf{P R}^{\mathrm{b}}$ & Estadual/Regionais & 19 & 24 & 360 & - & 360 & - & - \\
\hline \multirow[t]{3}{*}{ Subtotal } & & 19 & 24 & 360 & - & 360 & - & - \\
\hline & Santa Maria & 29 & 1 & 21 & 179 & 2.000 & 179 & 95 \\
\hline & Santa Cruz do Sul & 9 & 18 & 180 & 60 & 629 & 60 & 102 \\
\hline \multirow[t]{4}{*}{$\mathbf{R S}^{\mathrm{c}}$} & Ijuí & 1 & 2 & 63 & 5 & 50 & - & 28 \\
\hline & Passo Fundo & 1 & 1 & 38 & - & - & - & - \\
\hline & Porto Alegre & 1 & 2 & 31 & 1 & - & - & 8 \\
\hline & Pelotas & 1 & 2 & 24 & - & - & - & - \\
\hline Subtotal & & 42 & 26 & 357 & 245 & 2.679 & 239 & 233 \\
\hline $\mathrm{RJ}^{\mathrm{d}}$ & & 6 & - & - & - & - & - & 131 \\
\hline Subtotal & & 6 & - & - & - & - & - & 131 \\
\hline $\mathrm{SC}^{\mathrm{e}}$ & Estadual/Regionais & 91 & - & - & 621 & 9.315 & - & - \\
\hline Subtotal & & 91 & - & - & 621 & 9.315 & - & - \\
\hline \multirow[t]{2}{*}{$S P^{f}$} & Campinas & - & - & - & 112 & 200 & 10 & 200 \\
\hline & Estadual/Regionais & - & - & - & 12 & 1.075 & - & - \\
\hline Subtotal & & 22 & 85 & - & 124 & 1.275 & 10 & 200 \\
\hline Total & & 193 & 158 & 351 & 1154 & 16.089 & 431 & 564 \\
\hline
\end{tabular}

Fonte: Relatório de vigilância. BA, PR, RS, RJ, SC, SP, 2014.

$\mathrm{BA}=$ Bahia. $\mathrm{PR}=$ Paraná. $\mathrm{RS}=$ Rio Grande do Sul. $\mathrm{RJ}=$ Rio de Janeiro. SC = Santa Catarina. SP = São Paulo.

$\mathrm{PRC}=$ Postos de Revenda de Combustíveis. Trab = Trabalhadores. VISAT = VISAT. Centro de Referência em Saúde do

Trabalhador. Período de tempo vigilâncias: ${ }^{\mathrm{a}}\left(2004\right.$-2014), ${ }^{\mathrm{b}}\left(2009\right.$-2012), ${ }^{\mathrm{c}, \mathrm{d}, \mathrm{e}}\left(2010\right.$-2014), ${ }^{\mathrm{f}}(2008-2014)$. Período de tempo assistência: ${ }^{c, d}(2010-2013),{ }^{f}(2009-2010)$.

\section{A ação integrada de VISAT e VISAM em PRC}

Em São Paulo ${ }^{14}$, onde o critério foi a gravidade da exposição pela seleção de áreas contaminadas e seus fatores ambientais de riscos à saúde, as análises dos órgãos ambientais apontam para um contingente de $53 \%(\mathrm{n}=4.771)$ de áreas contaminadas. Dessas, 75\% $(\mathrm{n}=3.597)$ apresentam passivos de postos de distribuição de combustíveis e $52 \%(\mathrm{n}=2.481)$ estão localizadas em regiões metropolitanas. Os resultados das VISAT e VISAM evidenciam problemas referentes ao armazenamento de combustíveis, estoques de produtos químicos e escapamentos de gás ou vapor inflamável, de forma contínua ou intermitente, durante as operações dos equipamentos dos postos. Além disso, agravam o potencial de contaminação questões referentes às instalações, com sistemas de manutenção inadequados ou insuficientes, instalações obsoletas, ausência ou uso inadequado de equipamentos para a detecção de vazamento, entre outros. Essas situações, pelo potencial poluidor e gerador de acidentes, transformam-se em fontes de ocorrência de derramamentos, vazamentos, contaminação de água, solo, ar, incêndios e explosões. 


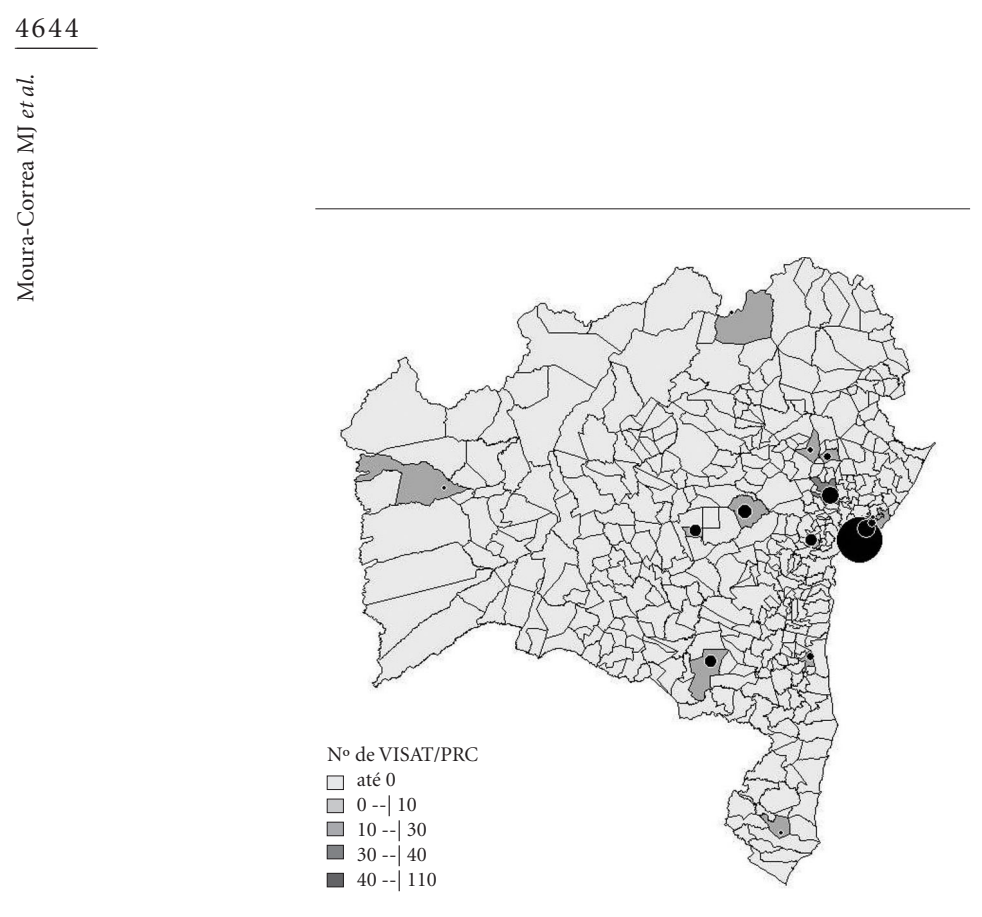

Figura 1. Distribuição do número de Postos de Revenda de Combustíveis vistoriados, por município. Bahia 2004 a 2014

Fonte: Relatório de vigilância. Bahia, 2014.

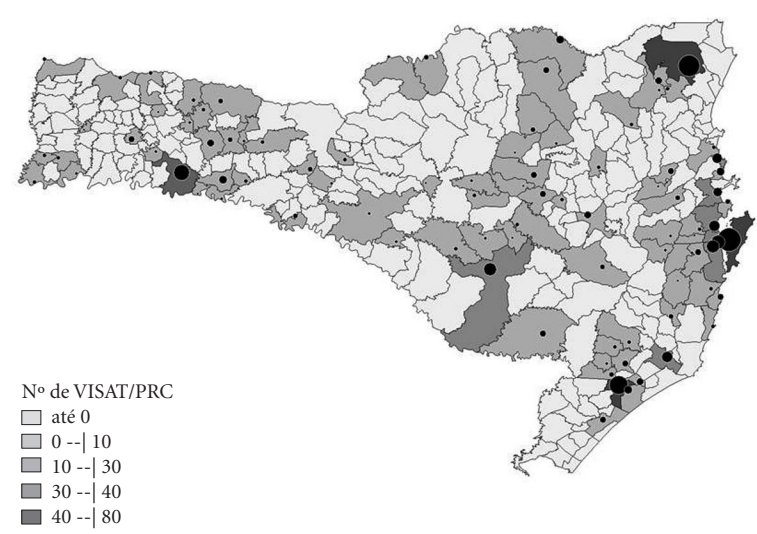

Figura 2. Distribuição do número de Postos de Revenda de Combustíveis vistoriados, por município. Santa Catarina 2010 a 2014.

Fonte: Relatório de vigilância. Santa Catarina, 2014.

\section{Os tipos de abordagens da VISAT}

O método de VISAT instituído contempla dois eixos de atuação: 1. ação direta de VISAT; e 2 ação integrada de VISAT e estudos/pesquisa.
No primeiro grupo, estão os estados da Bahia, Paraná, São Paulo e Santa Catarina, que também apresentam especificidades em sua atuação de VISAT. Na Bahia, por exemplo, foram realizados mapeamentos de risco com intervenções em 187 PRC, abrangendo um total de 2.745 trabalhadores localizados em 23 municípios do Estado, sendo a maioria situada na Região Metropolitana de Salvador, entre 2004 a 2014. No Paraná, foram vistoriados aproximadamente 360 trabalhadores de 24 PRC em 19 municípios, com análise coletiva do trabalho sobre questões de saúde e ambiente em 5 trabalhadores, entre 2009 e 2012. Em Santa Catarina, a Coordenação do Cerest Estadual, em conjunto com os CEREST regionais, realizou mapeamento de risco em 623 PRC, abrangendo 9.315 trabalhadores de 91 municípios, no período de 2010 a 2014. Em São Paulo, a VISAT abrangeu 22 municípios das regiões de Campinas, Grande ABC e Baixada Santista. Na fase piloto, foram inspecionados 85 postos com identificação dos riscos para os trabalhadores e avaliação da exposição da população em razão da área contaminada, que abrangeu em torno de 1.275 trabalhadores, entre 2008 e 2013. Em Campinas, foram inspecionados 10 PRC, sendo 8 em áreas contaminadas, e realizadas avaliações de saúde em 200 trabalhadores, com a análise de 1.276 exames do ácido trans, trans-mucônico em trabalhadores de 112 PRC (Tabela 1).

No segundo grupo, com formato de atividades de ensino, pesquisa e VISAT, encontram-se o Rio Grande do Sul e o Rio de Janeiro, cujas ações foram desencadeadas entre 2009 e 2014. No Rio Grande do Sul, o projeto coordenado pela Divisão de VISAT tem uma proposta de ensino e pesquisa-ação enquanto um método articulador da formação em serviço, ao integrar a produção do conhecimento e a intervenção no âmbito da VISAT em PRC. Nesse estado, a pesquisa e ação se estruturou pela abordagem interinstitucional e intrasetorial com a Universidade Federal do Rio Grande do Sul (UFRGS), o Laboratório Central do Estado, VISAT e VISAM e os CEREST regionais. As vigilâncias dos ambientes de trabalho abrangeram um total de 271 PRC, com 3.036 trabalhadores de 42 municípios, dos quais foram avaliados 233 trabalhadores (Tabela 1). Os resultados da avaliação dos trabalhadores revelaram alterações hematológicas em marcadores de função hepática e renal, prejuízo auditivo e dos indicadores de dose interna (ácido trans, trans -mucônico e fenil-mercaptúrico) ${ }^{19,23}$. No Rio de Janeiro, a partir da coordenação do Cesteh/ Ensp/Fiocruz, também por meio de um projeto 
interinstitucional com o Instituto Nacional do Câncer, Secretaria Municipal de Saúde do Rio de Janeiro e CEREST/RJ, os quais realizaram estudos, foram avaliados 131 trabalhadores de 6 PRC da Zona Norte da capital (Tabela 1). O estudo avaliou o grupo de trabalhadores, identificando alterações nos indicadores de genotoxicidade e susceptibilidade ${ }^{24}$.

Os relatórios de inspeções nos $\mathrm{PRCs}^{14-20}$ evidenciaram que nestes ambientes de trabalho são realizadas atividades de risco à saúde e que os trabalhadores com maior exposição aos hidrocarbonetos aromáticos são os frentistas, chefes de pista, encarregados de medição e descarga de combustíveis e o responsável pelos testes de qualidade. Contribuem para a perpetuação desta exposição ocupacional a pouca cultura prevencionista à saúde e segurança e a ausência de equipamentos de captação dos vapores no ambiente e processo de trabalho.

\section{Considerações finais}

A experiência de vigilância da exposição ao benzeno e intervenção descentralizada nos estados, além dos resultados diretos aos trabalhadores, à população do entorno e ao meio ambiente, representou importante modelo de ação multidisciplinar em rede intrainstitucional e interinstitucional integrada, por meio de organização articulada dos serviços do SUS para o desenvolvimento da VISAT e VISAM. Demonstra a potencialidade da VISAT em rede pelo estabelecimento de interações entre as instituições públicas de ensino e pesquisa e as instâncias organizativas da sociedade civil para uma prática integrada do controle e monitoramento das exposições do benzeno nos ambientes de trabalho dos PRCs. A VISAT se operacionaliza pelas modalidades de intervenção sobre o risco, formação para a vigilância, pesquisas e estudos, avaliação clínicas e laboratoriais, avaliação da exposição e efeitos tóxicos, genotóxicas e genéticos dos trabalhadores, associadas às ações de promoção, assistência e monitoramento dos expostos e da exposição. Todos esses componentes constituem etapas que integram a totalidade da vigilância em áreas de atuação dos CEREST sob os ambientes e indivíduos submetidos a essa exposição ocupacional. Sua estrutura metodológica é resultado dos perfis institucionais dos técnicos e pesquisadores que compõem a rede e do compromisso com as diretrizes e da VISAT no SUS.

Pelo potencial de impacto, essa modalidade de intervenção pode tornar-se uma possibilidade de operacionalização das demais vigilâncias ao risco químico no SUS, capaz de integrar conhecimento científico, investigação e práticas articuladas para a proteção da saúde dos trabalhadores e melhorias dos ambientes de trabalho. Esta ação de VISAT, entre outras experiências bem-sucedidas, pode ser um exemplo de construção da atenção integral em saúde do trabalhador, objetivo da Política Nacional de Saúde do Trabalhador e da Trabalhadora do SUS.

No que tange a participação dos trabalhadores, a perspectiva de atuação por ramo de atividade econômica é uma estratégia positiva, na medida em que segue a lógica da organização dos trabalhadores, viabilizando a participação sindical na sua base com a priorização, delimitação e acompanhamento das ações de vigilância.

A experiência construída de VISAT em rede demonstra um percurso na construção de ações com temporalidades e intensidades diversas. Evidencia possibilidades múltiplas de interações institucionais com suas dinâmicas e focos complementares, ao configurar um processo de construção de um mosaico de intervenções em situações de risco do trabalho nos postos de combustíveis ${ }^{32}$

A comunicação e a consolidação de redes de visualização das múltiplas experiências demonstram a potencialidade da intervenção pela integração de esforços e expertises regionais e vêm ao encontro da consolidação desta ação como um programa nacional de VISAT em PRC.

Em síntese, a VISAT em PRC organiza a ação sanitária integrando as vigilâncias epidemiológica, ambiental e sanitária com ações assistenciais e interinstitucionais com órgãos do setor ambiental e do trabalho, interagindo com sindicatos de trabalhadores e patronais em um processo de negociação e intervenção contínua.

Entretanto, apesar destes importantes avanços, também persistem desafios que ainda precisam ser equacionados, especialmente no que se refere ao aperfeiçoamento de instrumentos e métodos de investigação da exposição ocupacional e implementação de medida de controle e restrição da exposição ocupacional e ambiental, bem com o estabelecimento de educação permanente do grupo técnico e definição estrutural e organizacional para a integração das equipes de trabalho em rede para fins de processo contínuo da vigilância.

Desenvolve-se, assim, a partir desta experiência articulada em rede de VISAT em PRC no Brasil, uma prática orientada por atividades de complexidades técnicas distintas em suas múltiplas 
interações locais, que convergem para o mesmo objeto com a adoção da base operacional por ramo produtivo. Pelas características integradas e complementares, esta modalidade de ação supera as limitações normativas verticais, geralmente presentes em ações de âmbito nacional, assim como a dicotomia entre as práticas individuais e coletivas. Representa importante perspectiva de ações articuladas de Vigilância no SUS e de organização dos serviços para ampliação das dimensões e dos territórios, que se efetiva pela integração da formação, pesquisa-ação, vigilância e assistência, transformando-se em um processo de interconexão entre o conhecimento e a práxis participativa entre técnicos, trabalhadores e pesquisadores para a vigilância da exposição ao benzeno nos ambientes de trabalho e no meio ambiente.

\section{Colaboradores}

MJ Moura-Correa, AJR Jacobina, AS Santos, RDC Pinheiro, MAC Menezes, AM Tavares e NF Pinto participaram do desenvolvimento do trabalho, elaboração e revisão do manuscrito.

\section{Agradecimentos}

Aos trabalhadores dos PRC, técnicos das VISA, VISAM, VISAT e CEREST dos estados da BA, PR, RS, SC e SP e técnicos e pesquisadores do RJ, que participaram e contribuíram para a efetividade desta vigilância descentralizada.

Ao professor Jorge Mesquita Huet Machado, que coordenou, em conjunto com outros colegas, este processo de vigilância em rede e revisou este artigo. 


\section{Referências}

1. Weisel CP. Benzene exposure: An overview of monitoring methods and their findings. Chem Biol Interact [serial on the Internet] 2010 Jan [cited 2014 Ago 10]; 184: (1-) [about 10 p.]. Available from: http://www. ncbi.nlm.nih.gov/pubmed/20056112

2. Agency for Toxic Substances Disease Registry. ASTDR. Toxicological profile for benzene. U.S. Departament of Health and Human Services (DHHS). Public Health Service (PHS). [serial on the Internet] 2007 Aug [cited 2014 Ago 10]; [about 438p.]. Available from: http:// www.atsdr.cdc.gov/toxprofiles/tp3.html

3. Brasil. Ministério da Saúde (MS). Portaria no 3.120 de $1^{\circ}$ de julho de 1998. Aprova a Instrução Normativa de VISAT no Sistema Único de Saúde. Diário Oficial da União 1998; 2 jul.

4. Brasil. Ministério da Saúde (MS). Portaria GM/MS $\mathrm{n}^{\circ} 1.679$, de 19 de setembro de 2002. Dispõe sobre a estruturação da Rede Nacional de Atenção Integral à Saúde do Trabalhador no SUS e dá outras providências. Diário Oficial da União 2002; 20 set.

5. Brasil. Ministério da Saúde (MS). Portaria GM/MS no 776 , de 28 de abril de 2004. Dispõe sobre a regulamentação dos procedimentos relativos à vigilância da saúde dos trabalhadores expostos ao benzeno, e dá outras providências. Diário Oficial da União 2004; 29 abr.

6. Brasil. Ministério do Trabalho e Emprego. Secretaria de Inspeção do Trabalho. Portaria no 252, de 04 de agosto de 2011. Constitui a Subcomissão de Postos de Revendedores de combustíveis. Diário oficial da União 2011; 4 ago.

7. Paim JS. Modelos assistenciais: reformulando o pensamento e incorporando a proteção e a promoção da saúde. Notas para discussão nos Seminários Temáticos Permanentes. 2001 Mar [acessado 2014 set 17]; [cerca de 16 p.]. Disponível em: https://www.repositorio.ufba. be/ri/handle/ri/6168

8. Paim JS. Vigilância da Saúde: dos modelos assistenciais para a promoção da saúde. In: Czeresnia D, Freitas CM, organizadores. Promoção da Saúde: conceitos, reflexões e tendências. Rio de Janeiro: Fiocruz; 2003. p. 161-174.

9. Machado JMH. A proposito da VISAT. Cien Saude Colet $2005 ; 10(4): 987-992$.

10. Costa MFB. Estudo da aplicabilidade do ácido transtrans -mucônico urinário como indicador biológico de exposição ao benzeno [tese]. Rio de Janeiro: Escola Nacional de Saúde Pública; 2001.

11. Moura-Correa MJ. Prevalência da exposição ocupacional ao benzeno e mortalidade por leucemia entre os expostos: estimativas para o Brasil [tese]. Salvador: Universidade Federal da Bahia; 2014.

12. Instituto Brasileiro de Geografia e Estatística (IBGE). Censo Demográfico 2010 - Resultados gerais da amostra. Rio de Janeiro: IBGE; 2010.

13. Agência Nacional do Petróleo, Gás Natural e Biocombustíveis (ANP). Anuário estatístico brasileiro do petróleo, gás natural e biocombustíveis. Rio de janeiro: ANP; 2013.

14. São Paulo. Secretaria Estadual de Saúde. Divisão de VISAT, do Centro de Vigilância Sanitária (DVST/CEVS). Relatório de Vigilância da Exposição ao Benzeno em Postos de Combustiveis. São Paulo: DVST/CEVS; 2014.
15. Bahia. Secretaria Estadual de Saúde. Centro Estadual de Saúde do Trabalhador. Divisão de VISAT (CESAT/ DVAST). Relatório de Vigilância da Exposição ao Benzeno em Postos de Combustiveis. Salvador: CESAT/ DVAST; 2014.

16. Paraná. Secretaria Estadual de Saúde. Centro Estadual de Saúde do Trabalhador (CEST). Relatório de Vigilância da Exposição ao Benzeno em Postos de Combustíveis. Curitiba: CEST; 2014

17. Santa Catarina. Secretaria Estadual de Saúde. Centro de Referencia em Saúde do Trabalhador. Diretoria de Vigilância Sanitária. Relatório de Vigilância da Exposição ao Benzeno em Postos de Combustíveis. Florianópolis: CEREST/DIVS; 2014

18. Campinas. Secretaria Municipal de Saúde. Centro de Referência Regional em Saúde do Trabalhador (CEREST). Relatório de Vigilância da Exposição ao Benzeno em Postos de Combustíveis. Campinas: CEREST; 2014.

19. Rio Grande do Sul. Secretaria da Saúde do Estado. Divisão de VISAT. Centro de Vigilância em Saúde do Rio Grande do Sul. DVST/CEVS. Relatório de Vigilância da Exposição ao Benzeno em Postos de Combustíveis. Porto Alegre: DVST/CEVS; 2014

20. Rio de Janeiro. Centro de Estudos da Saúde do Trabalhador e Ecologia Humana, Escola Nacional de Saúde Pública Sergio Arouca, Fundação Oswaldo Cruz. Relatório de Vigilância da Exposicão ao Benzeno em Postos de Combustíveis. Rio de Janeiro: CESTH/ENSP/Fiocruz; 2014.

21. Santos APL, Lacaz FAC. A Saúde do Trabalhador no SUS: contexto, estratégias e desafios. In: Minayo-Gomez C, Machado JMH, Pena P, organizadores. Saúde do trabalhador na sociedade brasileira contemporânea. Rio de Janeiro: Abrasco; 2011. p. 87-106.

22. Vasconcellos LCF, Almeida CVB, Guedes DT. VISAT: passos para uma pedagogia. In: Moura-Correa MJ, Pinheiro, TMM; Merlo, ARC, organizadores. VISAT no Sistema Único de Saúde: Teorias e Práticas. Belo Horizonte: COOPMED; 2013. p. 79-97.

23. Moro AM, Charão MF, Brucker N, Durgante MB, Bubols G, Goethel G, Fracasso R, Nascimento S, Bulcão R, Gauer B, Barth A, Bochi G, Moresco R, Gioda A, Salvador M, Farsky S, Garcia SC. Genotoxicity and oxidative stress in gasoline station attendants. Muta Res 2013; 754(1):63-70

24. Costa-Amaral IC, Carvalho LVB, Pimentel JNS, Castro VS, Vieria JA, Tabalipa MM, Santas DVS, Teixeira LR, Alves SR, Larentis AL, Fonseca ASA, Menezes MAC, Sarcinelli PN. Comet assay evaluation on exposed workers in gas stations sited in northern Rio de Janeiro city. 11th International Conference of Environmental Mutagens; 2013 Nov 3-8; Foz do Iguaçu, Paraná. Brasil, 2013.

25. Campos MAA. Teste cometa: validação do método e avaliação da exposição ocupacional ao benzeno presente na gasolina através dos biomarcadores de exposição e genotoxicidade [tese]. Belo Horizonte: Universidade Federal de Minas Gerais; 2013.

26. Santos M. Metamorfoses do espaço habitado. São Paulo: Hucitec; 1996.

27. Laurell AC, Noriega M. Processo de Produção e Saúde Trabalho e Desgaste Operário. São Paulo: Cebes, Hucitec; 1989. 
28. Santana VS, Ferrite S. Vigilância Epidemiológica em Saúde do Trabalhador. In: Moura-Correa MJ, Pinheiro TMM, Merlo ARC, organizadores. VISAT no Sistema Único de Saúde: Teorias e Práticas. Belo Horizonte: COOPMED; 2013. p 99-123.

29. Silva Junior JB. Reflexões sobre o processo de monitoramento na gestão das ações de vigilância em saúde. Cien Saude Colet 2013; 18(5):1220-1221.

30. Minayo MCS. Editorial. Saúde, trabalho e ambiente em pauta permanente. Cien Saude Colet 2011; 16(8):33583359.

31. São Paulo. Companhia Ambiental do Estado de São Paulo - CETESB. [acessado 2009 mar 3]; [cerca de 2 p.]. Disponível em: http://www.cetesb.sp.gov.br/

32. Machado JMH, Porto MFS. Promoção da saúde e intersetorialidade: a experiência da VISAT na construção de redes. Epid. Serv. Saúde 2003; 12(3):121-130.

Artigo apresentado em 29/08/2014

Aprovado em 29/08/2014

Versão final apresentada em 08/09/2014 